\title{
Comparison of hydrocephalus metrics between infants successfully treated with endoscopic third ventriculostomy with choroid plexus cauterization and those treated with a ventriculoperitoneal shunt: a multicenter matched-cohort analysis
}

\author{
Michael C. Dewan, MD, MSCI, ${ }^{1}$ Jaims Lim, BS, ${ }^{1}$ Stephen R. Gannon, BS, ${ }^{1}$ David Heaner, BS, ${ }^{2}$ \\ Matthew C. Davis, MD, MPH, ${ }^{3}$ Brandy Vaughn, RN, BSN, ${ }^{4-6}$ Joshua J. Chern, MD, PhD, ${ }^{2}$ \\ Brandon G. Rocque, MD, MS, ${ }^{3}$ Paul Klimo Jr., MD, MPH, ${ }^{4-6}$ John C. Wellons III, MD, MSPH, ${ }^{1}$ and \\ Robert P. Naftel, MD1 \\ 'Department of Neurosurgery, Vanderbilt University, Division of Pediatric Neurosurgery, Monroe Carell Jr. Children's Hospital \\ at Vanderbilt, Nashville, Tennessee; ${ }^{2}$ Department of Neurosurgery, Children's Healthcare of Atlanta, Georgia; ${ }^{3}$ Department of \\ Neurosurgery, University of Alabama at Birmingham, Alabama; and ${ }^{4}$ Semmes Murphey Clinic; ${ }^{5}$ Department of Neurosurgery, \\ University of Tennessee; and 'Le Bonheur Children's Hospital, Memphis, Tennessee
}

OBJECTIVE It has been suggested that the treatment of infant hydrocephalus results in different craniometric changes depending upon whether ventriculoperitoneal shunt (VPS) placement or endoscopic third ventriculostomy with choroid plexus cauterization (ETVICPC) is performed. Without an objective and quantitative description of expected changes to the infant cranium and ventricles following ETV/CPC, asserting successful treatment of hydrocephalus is difficult. By comparing infants successfully treated via ETVICPC or VPS surgery, the authors of this study aimed to define the expected postoperative cranial and ventricular alterations at the time of clinical follow-up.

METHODS Patients who underwent successful treatment of hydrocephalus at 4 institutions with either VPS placement or ETV/CPC were matched in a 3:1 ratio on the basis of age and etiology. Commonly used cranial parameters (including head circumference $[\mathrm{HC}], \mathrm{HC}$ z-score, fontanelle status, and frontooccipital horn ratio [FOHR]) were compared pre- and postoperatively between treatment cohorts. First, baseline preoperative values were compared to ensure cohort equivalence. Next, postoperative metrics, including the relative change in metrics, were compared between treatment groups using multivariate linear regression.

RESULTS Across 4 institutions, 18 ETV/CPC-treated and 54 VPS-treated infants with hydrocephalus were matched and compared at 6 months postoperatively. The most common etiologies of hydrocephalus were myelomeningocele $(61 \%)$, followed by congenital communicating hydrocephalus $(17 \%)$, aqueductal stenosis $(11 \%)$, and intraventricular hemorrhage $(6 \%)$. The mean age at the time of CSF diversion was similar between ETVICPC- and VPS-treated patients (3.4 vs 2.9 months; $p=0.69)$, as were all preoperative cranial hydrocephalus metrics $(p>0.05)$. Postoperatively, the ventricle size FOHR decreased significantly more following VPS surgery $(-0.15)$ than following ETV/CPC $(-0.02)(p<0.001)$, yielding a lower postoperative FOHR in the VPS arm ( 0.42 vs $0.51 ; p=0.01)$. The HC percentile was greater in the ETV/ CPC cohort than in the VPS-treated patients (76th vs 54 th percentile; $p=0.046$ ). A significant difference in the postoperative z-score was not observed. With both treatment modalities, a bulging fontanelle reliably normalized at last follow-up. CONCLUSIONS Clinical and radiographic parameters following successful treatment of hydrocephalus in infants differed between ETV/CPC and VPS treatment. At 6 months post-ETV/CPC, ventricle size remained unchanged, whereas VPS-treated ventricles decreased to a near-normal FOHR. The HC growth control between the procedures was similar, although the final HC percentile may be lower after VPS. The fontanelle remained a reliable indicator of success for both

ABBREVIATIONS $C P C=$ choroid plexus cauterization; $E T V=$ endoscopic third ventriculostomy; $F O H R=$ frontooccipital horn ratio; $H C=$ head circumference; $I Q R=$ interquartile range; $\mathrm{MM}=$ myelomeningocele; VPS = ventriculoperitoneal shunt.

SUBMITTED July 27, 2017. ACCEPTED October 17, 2017.

INCLUDE WHEN CITING Published online February 2, 2018; DOI: 10.3171/2017.10.PEDS17421. 


\begin{abstract}
treatments. This study establishes expected cranial and ventricular parameters following ETV/CPC, which may be used to guide preoperative counseling and postoperative decision making.

https://thejns.org/doi/abs/10.3171/2017.10.PEDS17421
\end{abstract}

KEY WORDS choroid plexus cauterization; endoscopic third ventriculostomy; frontooccipital horn ratio; hydrocephalus; infant; z-score

$\mathrm{T}$ HE role of endoscopic third ventriculostomy with choroid plexus cauterization (ETV/CPC) in the management of infantile hydrocephalus continues to rapidly evolve as data worldwide accrue and are made available to the neurosurgical community. ${ }^{3}$ Throughout, the efficacy of ETV/CPC is necessarily tied to and compared with the treatment standard of ventricular shunting. ${ }^{13,15,19}$ Naturally, the goal of both procedures is to optimize neurological development by reducing intracranial pressure by means of diverting CSF from the intraventricular space, and, in the case of ETV/CPC, also by diminishing the production of CSF from the outset. Surgical success has traditionally been defined as the resolution of signs and symptoms of elevated intracranial pressure without the need for further surgical intervention.

However, although the surgical goal of both procedures is the same, the clinical picture of a successful outcome may differ. Following successful treatment of hydrocephalus via placement of a ventriculoperitoneal shunt (VPS), ventricular volume reliably decreases while head circumference (HC) trajectory decreases or plateaus. ${ }^{10,12}$ On the other hand, in a modest cohort of patients treated via ETV/ $\mathrm{CPC}$, we recently reported that nearly one-half of successfully treated patients will demonstrate persistent ventriculomegaly, and one-fifth of patients experience progressive macrocephaly. ${ }^{1}$ Others have demonstrated this phenomenon in older patients treated successfully for hydrocephalus via the more traditional ETV alone., ${ }^{5,6}$ The prospect of differing evaluation criteria depending upon the operation performed can make a surgeon's determination of treatment success or failure challenging. This is particularly challenging when the expected postoperative parameters have not been defined. In this study, by comparing infants successfully treated via ETV/CPC or VPS surgery, we sought to define the expected postoperative cranial and ventricular alterations at time of clinical follow-up.

\section{Methods}

\section{Study Design and Population}

This was a retrospective, matched-cohort study examining postoperative clinical metrics in patients successfully treated with ETV/CPC or a VPS. Institutional review board approval was obtained for this study. Between 2014 and 2016, 18 patients undergoing ETV/CPC for treatment of hydrocephalus at 2 institutions (Vanderbilt University Medical Center and University of Alabama at Birmingham) were prospectively enrolled in a pediatric hydrocephalus registry. Relevant demographic, clinical, and radiographic data were recorded from presentation until 6-month clinical follow-up, which has been established as a commonly used duration to classify treatment success or failure..$^{2,16,17}$ The authors' use of a flexible endoscope reflects what has been previously described in the infant hydrocephalus population. ${ }^{15}$ Of note, the goal to cauterize $>90 \%$ of the choroid plexus found within the lateral ventricles $^{9}$ was achieved in all 18 patients. Those who underwent successful treatment-and who could be successfully matched to 3 VPS-treated patients (see below) - constituted the ETV/CPC component of this study. Successful treatment is defined as the absence of either a second treatment for hydrocephalus or death due to hydrocephalus. ${ }^{8,15}$

\section{Matching VPS to ETVICPC}

A 3:1 ratio of matching VPS $(n=54)$ to ETV/CPC (n $=18$ ) was sought. Because both centers were primarily performing ETV/CPC on infants with hydrocephalus during this time period, 2 additional centers (where shunting was primarily used for treatment of infantile hydrocephalus) were recruited to reduce selection bias. For the VPS cohort, the following criteria were required for inclusion: primary VPS insertion, etiology matched to ETV/ CPC-treated patient, age matched ( \pm 2 months) to ETV/ CPC-treated patient, preoperative $\mathrm{HC}$ and fontanelle assessment documented, preoperative imaging sufficient to allow accurate calculation of frontooccipital horn ratio (FOHR), postoperative (between 4 and 8 months) $\mathrm{HC}$ and fontanelle assessment documented, and postoperative imaging sufficient to allow accurate calculation of FOHR.

Patients were excluded for any of the following reasons: VPS revision or ETV/CPC failure within 6 months of surgery, lack of sufficient preoperative or postoperative clinical evaluation (HC measurement, fontanelle assessment), or lack of sufficient imaging during preoperative or postoperative evaluation. Any patients successfully treated with ETV/CPC for whom 3 suitable VPS matches could not be identified were excluded.

\section{Clinical Metrics for ETV/CPC Outcome}

$\mathrm{HC}$ was obtained in the standard fashion at the bedside, using a disposable tape measure. ${ }^{14}$ Calculation of the $\mathrm{HC}$ $\mathrm{z}$-score and its specific use in infantile hydrocephalus has been previously described. ${ }^{1}$ Briefly, the z-score is an agecorrected, single-directional unit describing HC deviance; it is particularly useful for comparisons at the extremes of distribution. Values are based upon the standard provided by 2006 WHO Child Growth Standards (http://www.who. int/childgrowth/en/). As a reference, an $\mathrm{HC}$ z-score of -1 roughly correlates with the 15 th percentile, whereas a $z-$ score of 1 approaches the 85th percentile. Similarly, a zscore of -2 corresponds to the 2.3 percentile, and a score of 2 approaches the 98th percentile.

Ventricle size was measured by trained members of the research team using the FOHR, a validated ratio representing ventricular volume and shown to have high interobserver reliability in pediatric patients. ${ }^{11}$ Preopera- 
tive FOHR was measured with MRI or CT. MRI, ultrasound, and CT were used with variability at the discretion of the treating hospital/physician to measure postoperative FOHR. Although a uniform imaging modality was not used in this study, the interchangeability of several modalities with retained accuracy has been previously described..$^{11}$ The fontanelle quality was assessed by a neurosurgeon and documented in the medical record according to the institution's preferred verbiage. Upon retrospective extraction, fontanelle quality was then binned into 1 of 2 categories. A bulging fontanelle was defined as above the level of the surrounding external table of bone. Absence of a bulging fontanelle was denoted when the fontanelle was palpated at or below the level of the surrounding external table of bone (flat or concave, respectively). This definition has been found to be highly reliable. ${ }^{20}$

Postoperatively, patients underwent clinical evaluation at intervals determined by the treating surgeon. However, the clinical and radiographic parameters used for analysis were those obtained at the 6-month follow-up visit. First, after correction of age for prematurity, preoperative variables including age, fontanelle quality, $\mathrm{HC}$, and ventricular size were compared between ETV/CPC- and VPS-treated patients to ensure similarity. Next, from the postoperative period, the same hydrocephalus metrics were compared between the cohorts. In addition, the changes (value ${ }_{6 \text { _months }}$ - value $\left._{\text {preoperative }}\right)$ in $\mathrm{HC}, \mathrm{HC}$ percentile ( $\Delta \%$ ile), $\mathrm{HC}$ z-score $(\Delta \mathrm{z}$-score $)$, and FOHR $(\Delta \mathrm{FOHR})$ were also documented and compared between groups. A positive $\Delta \mathrm{z}$-score indicated an increase in the $\mathrm{HC}$ that was deviating further from the mean than the growth curve would otherwise project. A positive $\triangle \mathrm{FOHR}$ indicated an increase in the size of the ventricles. Naturally, a negative value of each $\Delta$ metric represented the opposite.

\section{Statistical Analysis}

Study data were collected separately by investigators at each individual site and managed using a shared Research Electronic Data Capture (REDCap) platform. ${ }^{4}$ Data analysis was performed in a de-identified manner using Stata software version 14 (StataCorp).

Data were summarized using counts and frequencies for categorical variables with relative proportions as percentages. Normally distributed descriptive data are given as the mean with standard deviation, and nonnormal skewed data are described using the median with interquartile range (IQR). Dichotomous data were compared between groups via the chi-square test, and comparisons of binary variables with fields containing $<5$ frequencies were compared using the Fisher's exact test. Postoperative cranial metric parameters were tested for significant associations by means of linear regression.

For each parameter, a simple, multivariate regression model was built to incorporate the target parameter of interest as the dependent variable; independent covariates included age (continuous), etiology (categorical), and surgical procedure (categorical). Accordingly, the beta coefficient and measure of significance for surgical procedure (ETV/CPC vs VPS placement) were recorded. Nonsignificant contribution of surgical procedure was then confirmed via t-test of surgical procedure for each model. Be- cause regression was used to determine the contribution of relevant variables to the variance in cranial metrics, no specific matching considerations were necessary to construct the model.

\section{Results \\ Patient Characteristics}

Seventy-two patients underwent surgery for hydrocephalus via ETV/CPC (18 patients) or VPS (54 patients). The patients were young infants, with a mean/median age of 3.0/0.9 months, and were nearly balanced for sex, with 33 males (46\%). By inclusion definition, all patients were deemed to have a successful surgical outcome at 6-month follow-up. The most common etiology was myelomeningocele (MM) (61\%), followed by congenital communicating hydrocephalus (17\%), aqueductal stenosis (11\%), intraventricular hemorrhage (6\%), and Dandy-Walker malformation (4\%) (Table 1). A parent or caretaker's concern for a bulging fontanelle (63\%) and enlarging head (63\%) was the most common reason for presentation for neurosurgical evaluation, whereas reports of eye abnormalities (10\%), vomiting (4\%), and apneic spells (4\%) were far less common in this infant population.

The 18 ETV/CPC-treated patients from 2 institutions (Vanderbilt University Medical Center and University of Alabama at Birmingham) were adequately matched in a 1:3 ratio with 54 VPS-treated patients from 3 institutions (University of Alabama at Birmingham, Le Bonheur Children's Hospital, and Emory University), according to the algorithm previously described. Matching by etiology was excellent for the $18 \mathrm{ETV} / \mathrm{CPC}$-treated patients; patient age at the time of CSF diversion was similar between ETV/ CPC- and VPS-treated patients (3.4 vs 2.9 months; $\mathrm{p}=$ 0.69). Preoperative cranial hydrocephalus metrics were similar between cohorts. Based upon surgeon physical examination findings, a bulging fontanelle was present in 17 (94\%) ETV/CPC-treated patients and 41 (76\%) VPStreated patients $(\mathrm{p}=0.165)$. HC absolute value, HC percentile, and $\mathrm{HC}$ z-score were all similar $(\mathrm{p}>0.3)$ between the cohorts prior to undergoing CSF diversion (Table 1). Ventricular size was also similar as measured by the median FOHR: 0.52 for ETV/CPC-treated patients and 0.56 for VPS-treated patients $(p=0.29)$.

\section{Postoperative Cranial Metrics: ETV/CPC Versus VPS}

All patients demonstrated resolution of symptoms and signs of persistent hydrocephalus at the time of last follow-up. Consistent with the study design, the mean time of follow-up assessment for ETV/CPC- and VPS-treated patients was 6.0 and 6.6 months, respectively $(\mathrm{p}=0.45)$. Multivariate linear regression accounted for age and etiology, and a separate model was constructed for each hydrocephalus metric of interest. Postoperatively, neither the change in HC $(p=0.749)$ nor the absolute HC values at 6 months $(p=0.161)$ differed between groups.

However, a difference in postoperative $\mathrm{HC}$ percentiles was observed. ETV/CPC-treated patients demonstrated a mean HC percentile of 76 compared with VPS-treated patients at a mean percentile of $54(\mathrm{p}=0.046)$. Although the average drop in $\mathrm{HC}$ percentile was calculated to be 12 
TABLE 1. Baseline comparison of preoperative variables between patients undergoing ETV/CPC or VPS insertion

\begin{tabular}{|c|c|c|c|}
\hline \multirow[b]{2}{*}{ Variable } & \multicolumn{2}{|c|}{ Procedure } & \multirow{2}{*}{$\begin{array}{c}p \\
\text { Value }\end{array}$} \\
\hline & ETV/CPC & VPS & \\
\hline No. of patients & 18 & 54 & \\
\hline Mean age in mos \pm SD & $3.4 \pm 4.1$ & $2.9 \pm 4.4$ & 0.69 \\
\hline Etiology of hydrocephalus, no. (\%) & & & NA \\
\hline Myelomeningocele & $11(61)$ & $33(61)$ & - \\
\hline Intraventricular hemorrhage & $1(6)$ & $3(6)$ & - \\
\hline Aqueductal stenosis & $2(11)$ & $6(11)$ & - \\
\hline Congenital communicating & $3(17)$ & $9(17)$ & - \\
\hline Other* & $1(6)$ & $3(6)$ & - \\
\hline Convex fontanelle, no. (\%) & $17(94)$ & $41(76)$ & 0.165 \\
\hline \multicolumn{4}{|l|}{$\mathrm{HC}$, median (IQR) } \\
\hline Centimeters & $41.0(39.0-45.5)$ & $40.8(36.3-45.5)$ & 0.33 \\
\hline Percentile & $94(63-99)$ & $88(26-99)$ & 0.31 \\
\hline z-score & $1.6(0.3-2.6)$ & $1.2(-0.6$ to 2.8$)$ & 0.64 \\
\hline FOHR, median (IQR) & $0.52(0.49-0.55)$ & $0.56(0.48-0.64)$ & 0.29 \\
\hline \multicolumn{4}{|l|}{ Presenting symptoms/signs, no. (\%) } \\
\hline Emesis & $0(0)$ & $3(6)$ & 0.57 \\
\hline Lethargy & $0(0)$ & $1(2)$ & 1.0 \\
\hline Irritability & $1(6)$ & $2(4)$ & 1.0 \\
\hline Delayed milestones & $1(6)$ & $1(2)$ & 0.44 \\
\hline Bulging fontanelle & $16(89)$ & $29(54)$ & 0.01 \\
\hline Enlarging head & $14(77)$ & $31(57)$ & 0.16 \\
\hline Upgaze palsy/paresis & $3(17)$ & $4(7)$ & 0.36 \\
\hline Apnea/bradycardia & $0(0)$ & $3(6)$ & 1.0 \\
\hline
\end{tabular}

points greater among patients who underwent VPS surgery, this difference was not statistically significant $(\mathrm{p}=$ $0.197)$. Neither the absolute postoperative z-score (1.53 vs $0.38 ; \mathrm{p}=0.098)$ nor the change in $\mathrm{z}$-score $(-0.21$ vs -1.04 ; $\mathrm{p}=0.166$ ) was different between the ETV/CPC and VPS treatment groups (Table 2).

Ventricle size decreased significantly more following VPS treatment than ETV/CPC. Specifically, the FOHR decreased by 0.15 in the VPS cohort, whereas patients who underwent ETV/CPC experienced negligible volume reduction $(-0.02)(\mathrm{p}<0.001)$. Accordingly, the absolute postoperative FOHR was less in the VPS group (0.42) than in the ETV/CPC group $(0.51)(\mathrm{p}=0.01)$. Finally, the vast majority of patients $(97 \%)$ with a bulging fontanelle were observed to have a flat or sunken fontanelle at the time of last follow-up, irrespective of the type of surgical intervention. Two patients who underwent shunting maintained a fontanelle characterized as above the bone at the 6-month follow-up mark. Among the 4 quantitative hydrocephalus metrics (HC, HC percentile, z-score, and FOHR), there were no significant differences observed among the 4 hospitals ( $p>0.147)$.

\section{Discussion}

In this study, postoperative cranial metrics following successful ETV/CPC or VPS treatment of hydrocephalus were compared in a matched-cohort analysis. Ventricular size was greater following ETV/CPC than after VPS placement; in the latter group, a 27\% reduction in FOHR was observed, whereas in patients who underwent ETV/ CPC no significant reduction in ventricle size was demonstrated. The absolute change in $\mathrm{HC}$ and change in $\mathrm{HC}$ percentile were similar between the treatment modalities. The final postoperative $\mathrm{HC}$ percentile, however, was somewhat higher in patients treated via ETV/CPC than in those treated via a VPS (76th vs 54th percentile, respectively). A bulging fontanelle reliably normalized following both procedures. Hydrocephalus cranial parameters of successfully treated infants are similar across treating hospitals.

ETV success has been studied extensively, including expectations for resolution of symptoms and change in ventricle size. ${ }^{5,6}$ Most of these studies included primarily older children undergoing ETV alone. The present study is unique for 2 reasons. First, ETV/CPC is a different procedure than ETV alone, and assumptions cannot be made that the response would be similar. Second, most studies on ETV, with few exceptions, do not include infants. These younger patients are unique in terms of means for their assessment. While older children may be assessed for resolution of symptoms and imaging changes, infants can be additionally assessed for head measurement and 
TABLE 2. Hydrocephalus metrics 6 months after successful ETV/CPC or VPS insertion

\begin{tabular}{|c|c|c|c|c|}
\hline \multirow[b]{2}{*}{ Metric } & \multicolumn{2}{|c|}{ Procedure } & \multirow[b]{2}{*}{ Coefficient $^{*}$} & \multirow{2}{*}{$\begin{array}{c}p \\
\text { value }\end{array}$} \\
\hline & ETV/CPC & VPS & & \\
\hline No. of patients & 18 & 54 & & \\
\hline $\mathrm{HC}$ in $\mathrm{cm}$, mean $\pm \mathrm{SD}$ & $46.3 \pm 3.0$ & $45.0 \pm 3.4$ & 1.02 & 0.161 \\
\hline$\Delta \mathrm{HC}$ in $\mathrm{cm}$, mean $\pm \mathrm{SD}$ & $3.9 \pm 3.2$ & $4.1 \pm 4.5$ & 0.318 & 0.749 \\
\hline $\mathrm{HC}$ percentile, mean $\pm \mathrm{SD}$ & $76.1 \pm 28.1$ & $54.4 \pm 38.0$ & 20.2 & 0.046 \\
\hline$\Delta \mathrm{HC}$ percentile, mean $\pm \mathrm{SD}$ & $-1.3 \pm 29.5$ & $-13.1 \pm 39.8$ & 13.7 & 0.197 \\
\hline z-score, mean \pm SD & $1.53 \pm 1.77$ & $0.38 \pm 2.38$ & 0.98 & 0.098 \\
\hline$\Delta z$-score, mean \pm SD & $-0.21 \pm 2.4$ & $-1.04 \pm 2.4$ & 0.904 & 0.166 \\
\hline Fontanelle change, no. (\%) & & & & NA \\
\hline Bulging to flat & $17(94)$ & $39(72)$ & & \\
\hline Flat to bulging & 0 & 0 & & \\
\hline Bulging to bulging & 0 & $2(4)$ & & \\
\hline Flat to flat & $1(6)$ & $13(24)$ & & \\
\hline FOHR, mean \pm SD & $0.51 \pm 0.08$ & $0.42 \pm 0.12$ & 0.09 & 0.01 \\
\hline$\Delta \mathrm{FOHR}$, mean $\pm \mathrm{SD}$ & $-0.02 \pm 0.06$ & $-0.15 \pm 0.11$ & 0.127 & $<0.001$ \\
\hline
\end{tabular}

fontanelle assessment, thus providing additional objective measurements to affirm success or failure. In addition, the rate of brain growth is highest during infancy, leading to unique intracranial dynamics that differ significantly from those of older children and adults after hydrocephalus treatment.

The stimulus for this study was derived from 2 clinical observations: 1) during follow-up, children with successful resolution of hydrocephalus following ETV/CPC appear different from those after VPS insertion; and 2) an inability to objectively quantify this difference makes postoperative clinical decision making challenging. By assigning expected norms following ETV/CPC to the infant patient (particularly in direct relation to VPS norms), surgeons may more objectively and more certainly judge treatment success, and advise patients and their families accordingly.

Establishing these craniometric differences between ETV/CPC and VPS treatment in infants corroborates data from previous studies on children treated via ETV alone. Nearly 2 decades prior, the modest influence of ETV alone on ventricle size was well documented. ${ }^{5,12}$ Nowosławska et al. demonstrated a significant difference in ventricle volume change between children treated via ETV and VPS..$^{10}$ In each of these studies, the mean patient age was several years older than in this study. In 2000, Kulkarni et al. evaluated postoperative ventricle size in children treated with ETV alone. ${ }^{6}$ Successfully treated patients showed a $16 \%$ reduction in ventricle size, relative to $7 \%$ in patients for whom treatment failed. Although this stands in contrast to our results, the mean patient age was 6.6 years-quite different from our young infant population.

Interestingly, in a relatively modest cohort of patients with MM, Warf et al. demonstrated no difference in ventricular volume (or cognitive outcomes) between ETV/ CPC and VPS surgery; however, there was a selection bias in treatment choice. ${ }^{18}$ In contrast, using a matched cohort, this study demonstrates a significant difference in the response of ventricle size. On average, patients successfully treated with ETV/CPC experience stabilization of their ventriculomegaly compared with shunted patients who experience a significant decrease in the size of their ventricles, nearly normalizing in size after 6 months (normal ventricle size is $0.37 \pm 0.026){ }^{11}$

Aside from the overt age difference, the etiologic makeup of our patient cohort deserves mention. Relative to other studies conducted in high-resource countries, $7,9,19$ the proportion of patients with MM was higher whereas the proportion with posthemorrhagic hydrocephalus was lower. Our etiologic proportions reflect the influence that etiology has on ETV/CPC success: MM-related hydrocephalus is associated with greater success via ETV/CPC than is posthemorrhagic hydrocephalus. ${ }^{1,9,16}$

Several strengths of this study are worth emphasizing. To our knowledge, it is the first effort to establish the expected postoperative cranial changes in infantile hydrocephalus by means of a direct comparison. Patients were carefully matched according to the 2 most important nonmodifiable determinants of ETV/CPC outcome: age and etiology (Riva-Cambrin J: ETV/CPC success and evolving indications and technique over time: an HCRN study, presentation at the Annual Meeting of the AANS/ CNS Section on Pediatric Neurological Surgery, Seattle, Washington, 2015). ${ }^{13}$ For this study in particular, the importance of these factors cannot be understated-cranial measurements directly correlate with patient age and are influenced by hydrocephalus etiology.

The effective bivariate matching lends the study validity and provides the framework from which the study's conclusions are derived. Moreover, by using multivariate linear regression to make comparisons between the treatment cohorts, potential differences in age matching were corrected for within the model. In addition, the multicenter 
design allows generalization beyond a single surgeon or hospital. Classically, shunt failure and now ETV/CPC failure are at risk for surgeon bias in making this determination. Although not completely eliminated, this bias is mitigated by involving multiple institutions and surgeons.

The observation that the postoperative $\mathrm{HC}$ percentile differs between ETV/CPC and VPS treatment, yet the preoperative values and the $\Delta$ values are similar, is curious. This probably reflects a power limitation; with more patients, a significantly larger $\Delta \mathrm{HC}$ percentile might be appreciated. An additional limitation is the absence of neurocognitive outcomes. At this time, no study in a Western society has made this comparison. Until long-term cognitive and quality-of-life outcomes are included, studies like this carry only indirect applicability to patient-centered clinical decision making.

Moreover, although we were careful to include the most commonly used and clinically accessible cranial metrics, a formal quantification of brain volume (and comparison thereof) was not done. Although this represents a relative limitation in terms of physiological understanding, an explanatory description was not among our study objectives. Instead, our primary goal was to describe the changes that a surgeon can expect following an evolving procedure (ETV/CPC) and how this compares with what he or she may be more familiar with (VPS surgery).

Ideally, neurocognitive outcomes between the procedures would guide the choice of therapy; however, until those exist, other indicators of success (including fontanelle status, head growth, and ventricle size) must be used. To summarize, ETV/CPC-treated patients should still be expected to have a sunken or flat fontanelle and appropriate head growth in relation to the growth curve, similar to shunting outcomes. However, these patients will have significantly larger ventricles, only stabilizing at the time of treatment.

\section{Conclusions}

Successful treatment of hydrocephalus in infants via ETV/CPC seems to be different than via VPS surgery. At 6 months post-ETV/CPC, ventricle size remained unchanged despite resolution of symptoms of hydrocephalus. Following VPS placement, however, the infant ventricles decreased to a near-normal FOHR. The absolute reduction in HC between the procedures was similar, although the final HC percentile may be lower after VPS surgery. This study establishes expected cranial and ventricular parameters following ETV/CPC, which may be used to guide preoperative counseling and postoperative decision making.

\section{Acknowledgments}

Research Electronic Data Capture (REDCap) use and management was funded by Institute for Clinical and Translational Research grant support (no. UL1 TR000445 from National Center for Advancing Translational Sciences/National Institutes of Health).

\section{References}

1. Dewan MC, Lim J, Morgan CD, Gannon SR, Shannon CN, Wellons JC III, et al: Endoscopic third ventriculostomy with choroid plexus cauterization outcome: distinguishing success from failure. J Neurosurg Pediatr 25:655-662, 2016

2. Dewan MC, Lim J, Shannon CN, Wellons JC III: The durability of endoscopic third ventriculostomy and ventriculoperitoneal shunts in children with hydrocephalus following posterior fossa tumor resection: a systematic review and time-to-failure analysis. J Neurosurg Pediatr 19:578-584, 2017

3. Dewan MC, Naftel RP: The global rise of endoscopic third ventriculostomy with choroid plexus cauterization in pediatric hydrocephalus. Pediatr Neurosurg 52:401-408, 2017

4. Harris PA, Taylor R, Thielke R, Payne J, Gonzalez N, Conde JG: Research electronic data capture (REDCap) - a metadata-driven methodology and workflow process for providing translational research informatics support. J Biomed Inform 42:377-381, 2009

5. Hopf NJ, Grunert P, Fries G, Resch KD, Perneczky A: Endoscopic third ventriculostomy: outcome analysis of 100 consecutive procedures. Neurosurgery 44:795-806, 1999

6. Kulkarni AV, Drake JM, Armstrong DC, Dirks PB: Imaging correlates of successful endoscopic third ventriculostomy. J Neurosurg 92:915-919, 2000

7. Kulkarni AV, Drake JM, Kestle JRW, Mallucci CL, Sgouros S, Constantini S: Endoscopic third ventriculostomy vs cerebrospinal fluid shunt in the treatment of hydrocephalus in children: a propensity score-adjusted analysis. Neurosurgery 67:588-593, 2010

8. Kulkarni AV, Drake JM, Mallucci CL, Sgouros S, Roth J, Constantini S: Endoscopic third ventriculostomy in the treatment of childhood hydrocephalus. J Pediatr 155:254-259, 259.e1, 2009

9. Kulkarni AV, Riva-Cambrin J, Browd SR, Drake JM, Holubkov R, Kestle JRW, et al: Endoscopic third ventriculostomy and choroid plexus cauterization in infants with hydrocephalus: a retrospective Hydrocephalus Clinical Research Network study. J Neurosurg Pediatr 14:224-229, 2014

10. Nowosławska E, Polis L, Kaniewska D, Mikołajczyk W, Krawczyk J, Szymański W, et al: Influence of neuroendoscopic third ventriculostomy on the size of ventricles in chronic hydrocephalus. J Child Neurol 19:579-587, 2004

11. O'Hayon BB, Drake JM, Ossip MG, Tuli S, Clarke M: Frontal and occipital horn ratio: a linear estimate of ventricular size for multiple imaging modalities in pediatric hydrocephalus. Pediatr Neurosurg 29:245-249, 1998

12. St George E, Natarajan K, Sgouros S: Changes in ventricular volume in hydrocephalic children following successful endoscopic third ventriculostomy. Childs Nerv Syst 20:834-838, 2004

13. Stone SSD, Warf BC: Combined endoscopic third ventriculostomy and choroid plexus cauterization as primary treatment for infant hydrocephalus: a prospective North American series. J Neurosurg Pediatr 14:439-446, 2014

14. Tanner J: Physical growth and development, in Forfar JO, Arneill GC (eds): Textbook of Paediatrics. Edinburgh: Churchill Livingstone, 1984, pp 278-330

15. Warf BC: Comparison of endoscopic third ventriculostomy alone and combined with choroid plexus cauterization in infants younger than 1 year of age: a prospective study in 550 African children. J Neurosurg 103 (6 Suppl):475-481, 2005

16. Warf BC, Campbell JW: Combined endoscopic third ventriculostomy and choroid plexus cauterization as primary treatment of hydrocephalus for infants with myelomeningocele: long-term results of a prospective intent-to-treat study in 115 East African infants. J Neurosurg Pediatr 2:310-316, 2008

17. Warf BC, Mugamba J, Kulkarni AV: Endoscopic third ventriculostomy in the treatment of childhood hydrocephalus in Uganda: report of a scoring system that predicts success. J Neurosurg Pediatr 5:143-148, 2010

18. Warf B, Ondoma S, Kulkarni A, Donnelly R, Ampeire M, 
Akona J, et al: Neurocognitive outcome and ventricular volume in children with myelomeningocele treated for hydrocephalus in Uganda. J Neurosurg Pediatr 4:564-570, 2009

19. Weil AG, Fallah A, Chamiraju P, Ragheb J, Bhatia S: Endoscopic third ventriculostomy and choroid plexus cauterization with a rigid neuroendoscope in infants with hydrocephalus. J Neurosurg Pediatr 17:163-173, 2016

20. Wellons JC III, Holubkov R, Browd SR, Riva-Cambrin J, Whitehead W, Kestle J, et al: The assessment of bulging fontanel and splitting of sutures in premature infants: an interrater reliability study by the Hydrocephalus Clinical Research Network. J Neurosurg Pediatr 11:12-14, 2013

\section{Disclosures}

The authors report no conflict of interest concerning the materi- als or methods used in this study or the findings specified in this paper.

\section{Author Contributions}

Conception and design: Dewan, Wellons, Naftel. Acquisition of data: Dewan, Lim, Gannon, Heaner, Davis, Vaughn, Chern. Analysis and interpretation of data: Dewan, Naftel. Drafting the article: Dewan. Critically revising the article: Dewan, Davis, Chern, Rocque, Klimo, Wellons, Naftel. Reviewed submitted version of manuscript: all authors. Approved the final version of the manuscript on behalf of all authors: Dewan. Statistical analysis: Dewan. Administrative/technical/material support: Gannon. Study supervision: Dewan, Rocque, Klimo, Wellons, Naftel.

\section{Correspondence}

Michael C. Dewan: Vanderbilt University, Nashville, TN. michael. dewan@vanderbilt.edu. 